\title{
Risk Evaluation of Electric Power Grid Investment in China Employing a Hybrid Novel MCDM Method
}

\author{
Yana Duan ${ }^{1}$, Yang Sun ${ }^{1}$, Yu Zhang ${ }^{1}$, Xiaoqi Fan ${ }^{1}$, Qinghuan Dong ${ }^{1}$ and Sen Guo ${ }^{2, *}$ \\ 1 National Electric Power Grid Inner Mongolia Eastern Electric Power Co., Ltd., Economic and Technological \\ Research Institute, Hohhot 010000, China; duanyana@md.sgcc.com.cn (Y.D.); sunyang@md.sgcc.com.cn (Y.S.); \\ zhangyu@md.sgcc.com.cn (Y.Z.); fanxiaoqi@md.sgcc.com.cn (X.F.); dongqinghuan@md.sgcc.com.cn (Q.D.) \\ 2 School of Economics and Management, North China Electric Power University, Beijing 102206, China \\ * Correspondence: guosen@ncepu.edu.cn or guosen324@163.com
}

check for updates

Citation: Duan, Y.; Sun, Y.; Zhang, Y.; Fan, X.; Dong, Q.; Guo, S. Risk Evaluation of Electric Power Grid Investment in China Employing a Hybrid Novel MCDM Method. Mathematics 2021, 9, 473. https:// doi.org/10.3390/math9050473

Academic Editor: James Liou

Received: 28 January 2021

Accepted: 20 February 2021

Published: 25 February 2021

Publisher's Note: MDPI stays neutral with regard to jurisdictional claims in published maps and institutional affiliations.

Copyright: (c) 2021 by the authors. Licensee MDPI, Basel, Switzerland. This article is an open access article distributed under the terms and conditions of the Creative Commons Attribution (CC BY) license (https:// creativecommons.org/licenses/by/ $4.0 /)$.
Abstract: Socio-economic development is undergoing changes in China, such as the recently proposed carbon peak and carbon neutral targets, new infrastructure development strategy and the Coronavirus disease 2019 (COVID-19) pandemic. Meanwhile, the new-round marketization reform of the electricity industry has been ongoing in China since 2015. Therefore, it is urgent to evaluate the risk of electric power grid investment in China under new socio-economic development situation, which can help the investors manage risk and reduce risk loss. In this paper, a hybrid novel multi-criteria decision making (MCDM) method combining the latest group MCDM method, namely, Bayesian best-worst method (BBWM) and improved matter-element extension model (IMEEM) is proposed for risk evaluation of electric power grid investment in China under new socio-economic development situation. The BBWM is used for the weights' determination of electric power grid investment risk criteria, and the IMEEM is employed to rank risk grade of electric power grid investment. The risk evaluation index system of electric power grid investment is built, including economic, social, environmental, technical and marketable risks. The risk of electric power grid investment under new socio-economic development situation in Inner Mongolia Autonomous Region of China is empirically evaluated by using the proposed MCDM method, and the results indicate that it belongs to "Medium" grade, but closer to "High" grade. The main contributions of this paper include: (1) it proposes a hybrid novel MCDM method combining the BBWM and IMEEM for risk evaluation of electric power grid investment; and (2) it provides a new view for risk evaluation of electric power grid investment including economic, social, environmental, technical and marketable risks. The proposed hybrid novel MCDM method for the risk evaluation of electric power grid investment is effective and practical.

Keywords: electric power grid investment (EPGI); risk evaluation (RE); bayesian best-worst method (BBWM); improved matter-element extension model (IMEEM); hybrid MCDM method (HMCDM)

\section{Introduction}

As a basic facility for national economic development, the electric power grid is responsible for ensuring the stable power supply of the whole society and promoting socioeconomic development [1,2]. Electric power grid investment is of great significance to the promotion of national economic development and energy transformation [3,4]. On the one hand, there is a long industry chain of the electric power grid investment and construction with large driving capacity, which can promote the development of front-end electrical equipment manufacturing, building materials and machinery manufacturing. Meanwhile, it can enhance the power supply capacity for electricity consumers and promote the production and living standards of electricity consumers [5]. On the other hand, the electric power grid investment has also played an important role in promoting clean energy transition [6]. There is regional disparity between electricity generation and electricity consumption in China. By conducting the electric power grid investment and building 
the electric power grid infrastructure, the renewable energy power generated in Northeast and Northwest regions can be transported to the electric power load center in Eastern China, which can tackle the issue of large-scale renewable energy allocation and increase the proportion of renewable energy power generation in total power generation $[7,8]$.

In March 2015, the policy document "Several Opinions on Further Deepening the Reform of Electric Power System" is promulgated, marking the official start of a new round of power system reform in China, which mainly includes a number of key reforms in the fields of the transmission and distribution prices, incremental distribution networks, and electricity sales market [9]. After the transmission and distribution price reform, the profit model of electric power grid company has changed from the traditional buying-selling gap to the transmission and distribution grid charge determined by the approved transmission and distribution price, which brings a great impact to the investment and operation of electric power grid companies [10]. The reforms of the incremental distribution network and the electricity sales market have also narrowed the profitability room of electric power grid companies, pushed them to reduce costs, and improved the efficiency and benefits of electric power grid investment [11]. Recently, the proposed carbon peak and carbon neutral targets, new infrastructure development strategy and the Coronavirus disease 2019 (COVID-19) pandemic have also posed great impacts on electric power grid investment in China. Therefore, the above-mentioned new socio-economic development environment will bring certain uncertainties and risks to the electric power grid investment in different ways. In the current socio-economic development environment, the electric power grid investment will face more risk factors, such as the risk of transmission and distribution price reform and uncertainty risk including nature disaster and COVID-19 pandemic. Therefore, it is urgent to evaluate the risk of electric power grid investment in the new socio-economic development environment.

In this paper, the risk evaluation of electric power grid investment in China in the new socio-economic development environment is conducted by employing a hybrid novel MCDM method. Compared with the existing research (please see details in Section 2), there are two main contributions in this paper:

(1) One contribution of this paper is to propose a hybrid novel generalized MCDM method for risk evaluation of electric power grid investment, combining the Bayesian best-worst method (BBWM) and improved matter-element extension model (IMMEM). The BBWM which can consider the preferences of multiple decision makers is employed to determine the weights of risk evaluation criteria of electric power grid investment, and the IMMEM is used to rank the overall risk grade of power grid investment. The BBWM and IMMEM are the first time to be employed for risk-related issue of electric power grid investment, which extend the application domains of these two MCDM methods.

(2) Another contribution is to provide a new view for risk evaluation of electric power grid investment, which not only includes economic and marketable risks, but also considers social, environmental and technical risks. The recent studies related to risk evaluation of electric power grid investment mainly focus on economic and marketable risk, so this paper extends the electric power grid investment risk scope which considers multiple sources of risks related to electric power grid investment. This paper comprehensively evaluates the risk of electric power grid investment considering economic risk, social risk, environmental risk, technical risk and marketable risk, which can fill the research gap in this field.

The rest of this paper is structured as follows: Section 2 conducts the literature reviews; Section 3 builds the risk evaluation index system for electric power grid investment under new socio-economic development situation; Section 4 introduces the proposed hybrid MCDM method including the BBWM and IMMEM for risk evaluation on electric power grid investment; the empirical analysis is conducted in Section 5 and the Section 6 discusses the results; Section 7 concludes this paper. 


\section{Literature Review}

Currently, there are some studies focusing on electricity power investment risk evaluation. Guindon AA and Wright DJ used the different economic viability measures to propose an analytical method to quantitatively assess risk of solar power projects considering the input parameters of interest [12]. Chebotareva G, et al. studied government-sponsored measures for renewable energy in Russia, conducted risk assessment in Russia's renewable energy projects, and modelled risk dynamics at all life cycle stages of renewable energy projects [13]. Sisodia GS, et al. conducted strategic business risk evaluation for sustainable energy investment in the United Arab Emirates (UAE) by using Monte Carlo simulations and net present value (NPV) methods [14]. Xu L, et al. evaluated the sustainable operationoriented investment risk for wind power in China by using the Monte Carlo method, and the optimization suggestions were also proposed for China's wind power projects [15]. Zhou S and Yang P identified the risk factors (including political, economic, social and technical risks) in distributed wind power in China based on literature reviews, and then conducted risk evaluation on distributed wind power by using Analytic Hierarchy Process (AHP) method [16]. Adiyeke E, et al. developed a time-consistent multi-stage stochastic optimization model for risk averse investment strategy of a private electricity generating company in a carbon constrained environment, which takes the minimization of conditional value at risk of the net present value as the objective [17]. Felling T, et al. used a partly meshed scenario tree to develop a methodology to evaluate the electricity generation company risk in extended central western Europe [18]. Wu YN, et al. assessed the renewable energy investment risk in belt and road initiative countries by using Analytic Network Process (ANP) and cloud model methods, and the sensitivity analyze for renewable energy investment decisions was also conducted [19]. Yuan JH, et al. assessed the coal-fired power plant investment risk for a serial of countries along the Belt and Road initiative by using the hybrid ANP-Entropy-TODIM (an acronym in Portuguese for interactive and multi-criteria decision making) method, and the results indicated Singapore has the lowest risk for China's coal-fired power plant investment [20]. Kul C, et al. proposed a multi-criteria decision methodology based three-stage decision framework to assess the renewable energy investment risk factors for sustainable development in Turkey by using Delphi, AHP and Fuzzy Weighted Aggregated Sum Product Assessment (FWASPAS) methods [21]. Yuan $\mathrm{JH}$, et al. conducted electric power investment risk assessment for Belt and Road initiative nations, proposed a nine-dimensional indicator system, and then evaluated the electric power investment risk of 21 countries by employing the entropy weighting method and fuzzy integrated evaluation model [22]. Egli F identified the five most relevant renewable energy technologies investment risk types based on 40 interviews in the United Kingdom, Germany and Italy, which include curtailment, policy, price, resource and technology [23]. Williams NJ, et al. assessed the investment risk of microgrid utilities for rural electrification, and identified the key uncertain variables influencing microgrid investments in Rwanda by using Stochastic Techno-Economic Microgrid Model (STEMM) [24]. Gregory J and Sovacool BK studied the financial risks related to electricity infrastructure in Kenya, Tanzania, and Mozambique by conducting a systematic review of 815 "peer reviewed" papers [25].

From the above literatures' reviews, it can be seen that most current studies focus on the investment risk evaluation of electricity power generation types, especially the renewable energy power generation. However, the studies related to the risk of electric power grid investment are rarely and inadequate. Therefore, this paper conducts the risk evaluation on electric power grid investment considering different sources of risks such as economic, social, environmental, technological and marketable risks. Meanwhile, the risk evaluation index system for electric power grid investment is built including multiple risk criteria. Therefore, considering these multiple risk criteria, a hybrid novel MCDM method is proposed for the risk evaluation of electric power grid investment. 


\section{Risk Evaluation Index System for Electric Power Grid Investment under New Socio-Economic Development Situation}

Nowadays, China's socio-economic development is facing new situations and changes. At the beginning of 2020, China proposed a national strategy for accelerating "new infrastructure", which identifies seven domains including 5G network, ultra-high voltage (UHV), new energy vehicle charging piles, intercity high-speed rail and intercity rail transit, big data centers, artificial intelligence, and industrial Internet. These seven domains have direct or indirect relations with the electric power grid investment. In May 2020, China proposed to "deepen supply-side structural reforms, give full play to China's super-large-scale market advantages and domestic demand potential, and build a new development pattern in which the domestic cycle and international cycle promote each other." On 22 September 2020, China's President Xi delivered an important speech at the General Debate of the Seventy-fifth United Nations General Assembly, which proposed that China will enhance the nationally determined contributions, adopt more powerful policies and measures, strive to reach carbon dioxide emissions peak by 2030, and try to achieve carbon neutrality by 2060. In the field of electric power, the transmission and distribution price reform has posed a great impact on the operation and investment of electric power grid companies. Through this transmission and distribution price reform, the business model and profit margins of electric power grid companies have become more transparent. The profit model of electric power grid company has changed from the traditional buying-selling gap to the transmission and distribution grid charge determined by the approved transmission and distribution price. With the continuous liberalization on the electricity sales market, the market competition will become increasingly intense, and this fierce market competition will bring great uncertainty and risk to the economy of capital-intensive electric power grid investment project. Meanwhile, the electric power grid investment is also facing more uncertain factors, including COVID-19 pandemic, temporary electricity price reduction policies and natural disasters such as freezing.

The reasonable and proper evaluation index system is vital for the accurate risk evaluation of electric power grid investment. The representative and key risk criteria need to be included in the risk evaluation index system, which can reflect the main characteristics of electric power grid investment risk. In this paper, the risk evaluation index system of electric power grid investment is built not only including economic and marketable risks criteria, but also considers social, environmental and technical risks criteria. Moreover, these five risk criteria include several risk sub-criteria, respectively. The detailed determination process for risk evaluation index system of electric power grid investment is as follows:

Firstly, the expert panel is set up, which includes five experts in the fields of electric power industry management and enterprise risk management. Of which, three experts are enterprise practitioners and two experts are academic professors. Secondly, according to the related academic literatures [12-25], the characteristics of new socio-economic development situation and expert panel discussion, the criteria for risk evaluation of electric power grid investment are determined which include economic risk criterion, social risk criterion, environmental risk criterion, technical risk criterion and marketable risk criterion. Thirdly, the initial sub-criteria for economic, social, environmental, technical and marketable risk criteria are determined according to the related academic literatures [12-25] and industrial reports. Fourthly, the expert panel are invited to review the initial risk sub-criteria, and then select more important ones related to economic, social, environmental, technical and marketable risk criteria based on their practical experience and professional knowledge, respectively. Finally, according to the comments and the selected more important risk subcriteria from the invited experts, the final sub-criteria are determined for risk evaluation of electric power grid investment.

The risk evaluation index system for electric power grid investment is shown in Figure 1, which includes five risk criteria and fifteen risk sub-criteria. The five risk criteria include economic risk criterion, social risk criterion, environmental risk criterion, technical risk criterion and marketable risk criterion. 


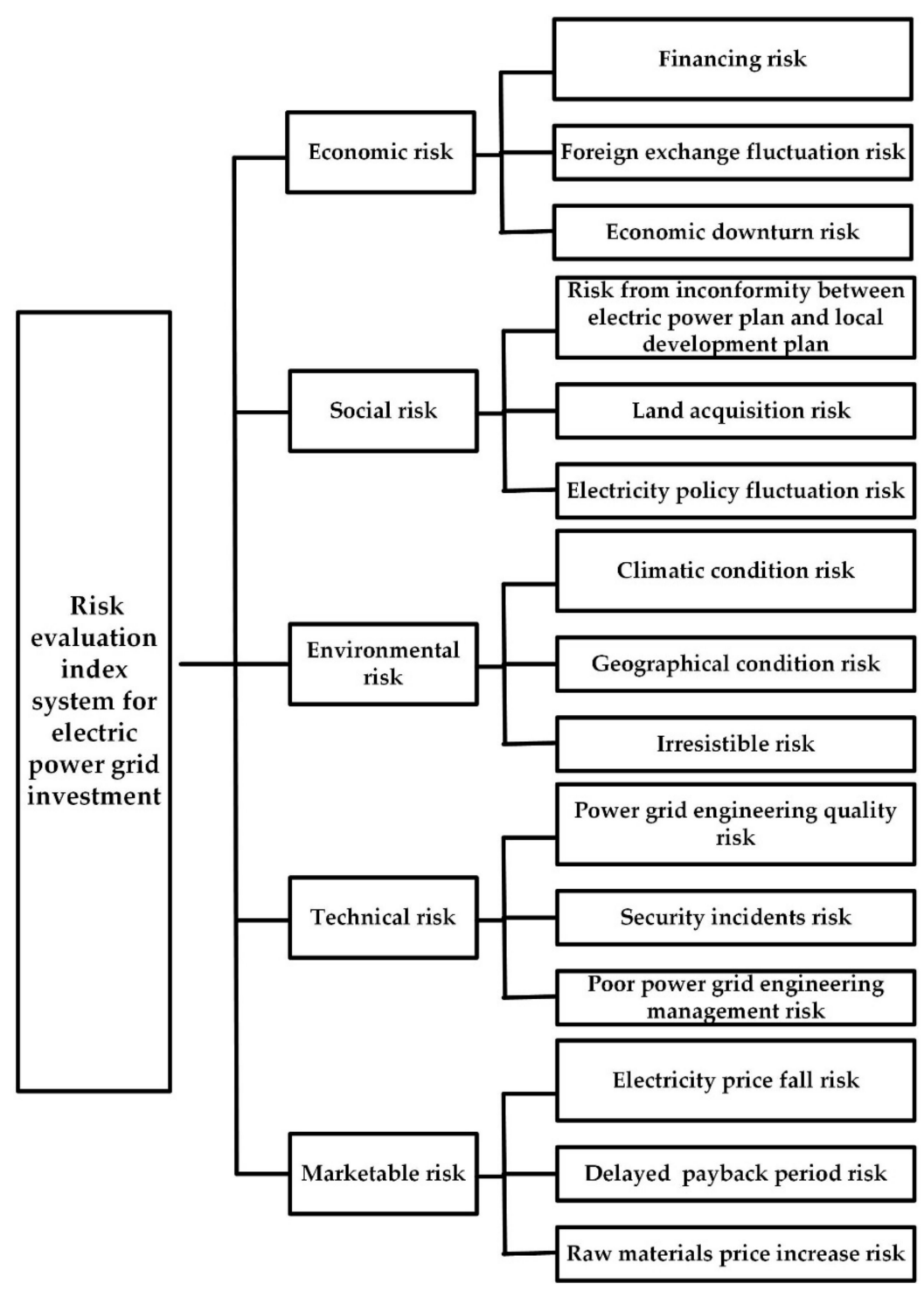

Figure 1. Risk evaluation index system for electric power grid investment.

For economic risk criterion, it includes financing risk sub-criterion (C1), foreign exchange fluctuation risk sub-criterion (C2), and economic downturn risk sub-criterion (C3).

For social risk criterion, it includes risk sub-criterion from inconformity between electric power plan and local development plan (C4), land acquisition risk sub-criterion (C5), and electricity policy fluctuation risk sub-criterion (C6).

For environmental risk criterion, it includes climatic condition risk sub-criterion (C7), geographical condition risk sub-criterion (C8), and irresistible risk (such as nature disaster and COVID-19) sub-criterion (C9).

For technical risk criterion, it includes power grid engineering quality risk subcriterion (C10), security incidents risk sub-criterion (C11), and poor power grid engineering management risk sub-criterion (C12).

For marketable risk criterion, it includes electricity price fall risk sub-criterion (C13), delayed payback period risk sub-criterion (C14), and raw materials price increase risk sub-criterion (C15).

\section{The Proposed MCDM Methodology for Risk Evaluation of Electric Power Grid Investment}

In this paper, the comprehensive risk of electric power grid investment is evaluated considering multiple risk criteria including economic risk, social risk, environmental 
risk, technical risk and marketable risk. Actually, there are high risks for some criteria of electric power grid investment, but other criteria may hold low risk. Therefore, the multiple risk criteria of electric power grid investment may be conflicting, which need to be simultaneously considered for comprehensive risk evaluation of electric power grid investment. The MCDM method, such as Matter-element Extension Model (MEEM), Technique for Order Preference by Similarity to Ideal Solution (TOPSIS), VlseKriterijumska Optimizacija I Kompromisno Resenje (VIKOR) and Preference Ranking Organization Method for Enrichment of Evaluations II (PROMETHEE II) [26-28], can consider conflicting criteria to make proper and effective decision, which has been used to solve many practical issues. Therefore, the MCDM method is employed to evaluate the risk of electric power grid investment, which is composed of the BBWM and IMEEM. The BBWM is employed to determine the weights of risk criteria of electric power grid investment, and the IMEEM is used to rank the comprehensive risk grade of electric power grid investment. The detailed theories of the hybrid MCDM method are introduced in the following.

\subsection{The Basic Theory of BBWM for Calculating Weights of Risk Criteria}

The Bayesian Best-Worst Method (BBWM) is proposed in 2019 [29], which is an extension of Best-Worst Method (BWM) proposed in 2015 [30]. The BBWM can consider different preferences of multiple decision makers simultaneously in probability distribution style other than mean operator method, which can avoid information loss in decision making process $[11,28]$. Therefore, the BBWM is superior to the BWM, especially in the case of multiple decision makers in decision-making process, namely group decision making.

The detailed step of the BWM is shown in Figure 2 [29]. The main drawback of the BWM is that it can only calculate the weights of decision-making criteria based on the preference of only one decision-maker [31]. If there are $n$ decision makers, the BWM needs to be conducted $n$ times, and then there will be $n$ weighting results for each criterion. To obtain the final weight of each criterion, the mean operator method needs to be used, but this method has drawbacks such as outlier sensitivity and restricted information provision [28]. To tackle this issue, the BBWM is proposed, which can determine the weight of each criterion at one time and not use mean operator method.

The BBWM have same inputs with the BWM, namely the "Best-to-Others" vector $A_{B}$ and "Others-to-Worst" vector $A_{W}$. However, the outputs of the BBWM are different from BWM, which include the optimal aggregated weights of decision-making criteria derived from more than one decision-makers and the confidence levels of criteria weights. For the BBWM, the probabilistic interpretations are employed to represent the inputs and outputs, and the inputs and outputs of BBWM are conducted as probability distributions with multinomial distribution. For the BBWM, the probability mass function for the multinomial distribution related to the worst criterion $A_{W}$ is:

$$
P\left(A_{W} \mid w\right)=\frac{\left(\sum_{j=1}^{n} a_{j W}\right) !}{\prod_{j=1}^{n} a_{j W} !} \prod_{j=1}^{n} w_{j}^{a_{j W}}
$$

where $w$ stands for the probability distribution; $a_{j W}$ is the significance of the $j$ th criterion to the worst criterion; and "!" is the factorial.

Then, the best criterion $A_{B}$ can also be modeled by employing the multinomial distribution. According to the principle of the BBWM, it can obtain:

$$
\begin{gathered}
\frac{w_{j}}{w_{W}} \alpha a_{j W} \\
\frac{w_{B}}{w_{j}} \alpha a_{B j}
\end{gathered}
$$

In the BBWM, the $w$ in the multinomial distribution is determined by employing the statistical inference technique. The maximum likelihood estimation method and the Dirichlet distribution are both used to weight the criteria in the Bayesian inference. Meanwhile, 
to solve the complexity issue for the maximum likelihood estimation inference, a Bayesian hierarchical model is employed, and the Markov-chain Monte Carlo method is used to obtain the posterior distribution of Bayesian hierarchical model. The detailed operation processes of these methods can consult Ref. [28]. In the BBWM, the probabilistic model is substituted for "Min-Max" rule in the BWM, and the credal orderings of criteria weights can also been obtained, which have been elaborated in Ref. [28].

As a latest group MCDM method, the BBWM shows priority and better performance over basic BWM, which can consider preferences of more than one decision makers at one time and avoid information loss by using probability distributions method.

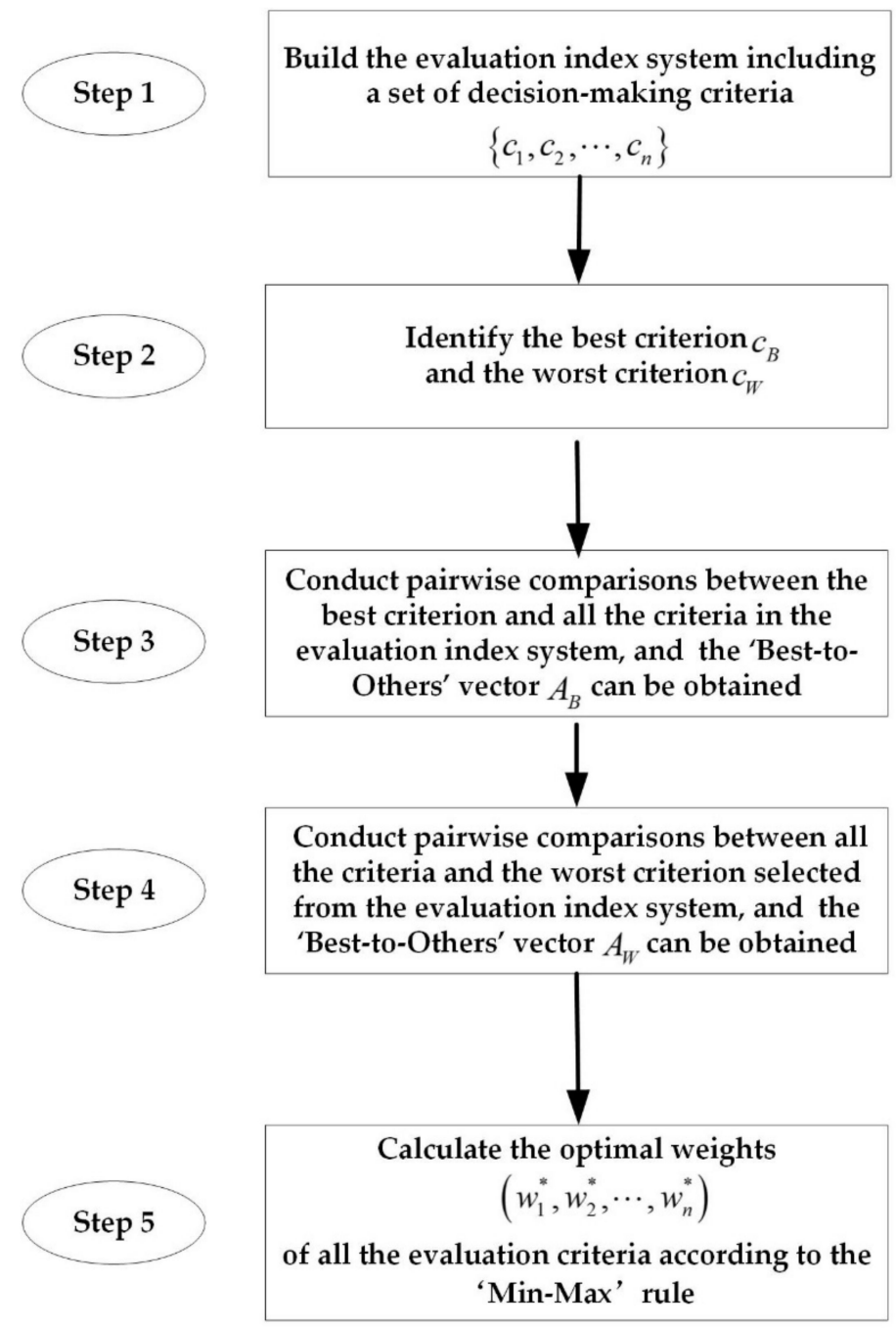

Figure 2. The detailed step of the BWM.

\subsection{The IMEEM for Risk Ranking of Electric Power Grid Investment}

The matter-element extension model proposed in 1983 can evaluate the object considering multiple conflicting criteria based on the correlation degrees between the evaluated object and the pre-set different evaluation levels, which is a common MCDM method and has been in many issues [32-34]. In the matter-element extension model, the evaluated object is named as the matter-element, representing by an ordered triple $R=(P, c, v)$. Of which, $P$ means the evaluated object; $c$ represents the characteristics of the evaluated object, which are the evaluation criteria; $v$ is the value of the evaluation criterion. Therefore, the matter-element (object) $P$ can be represented by Equation (4), which is described by $n$ characteristics (namely evaluation criteria) $C_{1}, C_{2}, \cdots, C_{n}$ and the corresponding values of the evaluation criteria $v_{1}, v_{2}, \cdots, v_{n}$. 


$$
R=(P, C, v)=\left[\begin{array}{ccc}
P & C_{1} & v_{1} \\
C_{2} & v_{2} \\
\ldots & \cdots \\
C_{n} & v_{n}
\end{array}\right]
$$

where $C=\left[C_{1}, C_{2}, \cdots, C_{n}\right]^{T}$ represents the characteristics of the evaluated object, namely multiple evaluation criteria, $v=\left[v_{1}, v_{2}, \cdots, v_{n}\right]^{T}$ is the corresponding values of multiple evaluation criteria $C$.

Many papers [35-38] have introduced the basic theory of the matter-element extension model, so it will not be elaborated in this paper in details. The matter-element extension model uses the maximum membership degree rule to evaluate the object according to the correlation degrees between the objective and different evaluation grades [39], but this rule may be easy to loss related evaluation information included in the evaluation criteria and cannot accurately reflect the fuzziness of the evaluated objective, which may lead to the biased evaluation ranking [11,40]. Therefore, the improved matter-element extension model will be employed in this paper, which the proximity degree rule is adopted to substitute for the maximum membership degree rule. It can tackle the above-mentioned issues and enhance the performance of the evaluation ranking.

The detailed steps of the IMEEM for risk ranking of electric power grid investment are as follows.

Step 1: Set the matter-element in the matter-element extension model.

The matter-element extension model includes three kinds of matter-elements, namely the matter-element in the classical field, the matter-elements in the controlled field, and the matter-elements to be evaluated. The matter-element in the classical field $R_{j}$ can be represented as:

$$
R_{j}=\left(P_{j}, C_{i}, v_{i j}\right)=\left[\begin{array}{ccc}
P_{j} & C_{1} & v_{1 j} \\
C_{2} & v_{2 j} \\
\ldots & \cdots \\
C_{n} & v_{n j}
\end{array}\right]=\left[\begin{array}{ccc}
P_{j} & C_{1} & \left\langle a_{1 j}, b_{1 j}\right\rangle \\
C_{2} & \left\langle a_{2 j}, b_{2 j}\right\rangle \\
\ldots & \ldots \\
C_{n} & \left\langle a_{n j}, b_{n j}\right\rangle
\end{array}\right]
$$

where $P_{j}$ represents the $j$ th risk grade of electric power grid investment; $C_{i}$ represents the characteristics (namely evaluation criteria) of the electric power grid investment; $v_{i j}$ represents the corresponding value of $C_{i}$ related to the $j$ th risk grade of electric power grid investment; $v_{i j}=\left\langle a_{i j}, b_{i j}\right\rangle(i=1,2, \cdots, n)$, and $a_{i j}, b_{i j}$ are lower boundary and upper boundary of the criterion value $v_{i j}$, respectively.

The matter-element in the controlled field $R_{p}$ can be presented as:

$$
R_{p}=\left(P, C_{i}, v_{i p}\right)=\left[\begin{array}{ccc}
P & C_{1} & v_{1 p} \\
& C_{2} & v_{2 p} \\
\ldots & \cdots \\
C_{n} & v_{n p}
\end{array}\right]=\left[\begin{array}{ccc}
P & C_{1} & \left\langle a_{1 p}, b_{1 p}\right\rangle \\
C_{2} & \left\langle a_{2 p}, b_{2 p}\right\rangle \\
\cdots & \ldots \\
& C_{n} & \left\langle a_{n p}, b_{n p}\right\rangle
\end{array}\right]
$$

where $P$ represents all the risk grades of electric power grid investment; $v_{i p}$ represents the value range of the $i$ th risk criterion $C_{i}$.

Step 2: Set the matter-element to be evaluated.

In this method, the matter-element to be evaluated is the research object, which is a certain electric power grid investment to be evaluated in this research. The matter-element to be evaluated $R_{0}$ can be represented as:

$$
R_{0}=\left(P_{0}, C_{i}, v_{i}\right)=\left[\begin{array}{ccc}
P_{0} & C_{1} & v_{1} \\
& C_{2} & v_{2} \\
\ldots & \cdots \\
& C_{n} & v_{n}
\end{array}\right]
$$


where $P_{0}$ represents the risk grade of a certain electric power grid investment to be evaluated; $v_{i}$ is the value of the risk criterion $C_{i}$ of a certain electric power grid investment to be evaluated.

Step 3: Determine the weights of risk criteria

The risk criteria weighting is quite vital for the comprehensive risk evaluation on electric power grid investment. According to the risk evaluation index system for electric power grid investment built in Section 3, all the risk criteria are qualitative criteria. Therefore, the BBWM is employed to determine the criteria weights of electric power grid investment.

Step 4: Calculate the proximity degrees related to different risk evaluation grades.

The detailed theoretical analysis of the proximity degree substituting for the correlation degrees can refer to Ref. [41], and the proximity degree function was proposed as:

$$
N=1-\frac{1}{n(n+1)} \sum_{i=1}^{n} D w_{i}
$$

where $N$ is the proximity degree; $n$ is the number of risk evaluation criteria; $D$ is the distance; and $w_{i}$ is the $i$ th risk criterion weight.

Further, Equation (9) gives the calculation method for the proximity degrees of a certain electric power grid investment related to different risk evaluation grades.

$$
N_{j}\left(p_{0}\right)=1-\frac{1}{n(n+1)} \sum_{i=1}^{n} D_{j}\left(v_{i}\right) w_{i}
$$

where $N_{j}\left(p_{0}\right)$ is the proximity degree of a certain electric power grid investment related to the $j$ th risk evaluation grade; $D_{j}\left(v_{i}\right)$ is the distance of a certain electric power grid investment from the matter-element in classical field, and $D_{j}\left(v_{i}\right)=\left|v_{i}-\frac{a_{i j}+b_{i j}}{2}\right|-\frac{1}{2}\left(b_{i j}-a_{i j}\right) ; n$ is the number of evaluation criteria.

Step 5: Risk evaluation ranking of electric power grid investment.

According to the calculated proximity degrees of electric power grid investment for different risk evaluation grades, it can be obtained that the risk of the electric power grid investment is belonged to the $j^{\prime}$ th risk evaluation grade based on Equation (10).

$$
N_{j^{\prime}}\left(p_{0}\right)=\max \left\{N_{j}\left(p_{0}\right)\right\}
$$

Suppose

$$
\bar{N}_{j}\left(p_{0}\right)=\frac{N_{j}\left(p_{0}\right)-\min _{j} N_{j}\left(p_{0}\right)}{\max _{j} N_{j}\left(p_{0}\right)-\min _{j} N_{j}\left(p_{0}\right)}
$$

where $\bar{N}_{j}\left(p_{0}\right)$ represents the standardized proximity degree of the electric power grid investment related to the $j$ th risk evaluation grade; $\max _{j} N_{j}\left(p_{0}\right)$ is the maximum of proximity degrees for all risk evaluation grades; and $\min _{j} N_{j}\left(p_{0}\right)$ is the minimum of proximity degrees for all risk evaluation grades.

$$
j^{*}=\frac{\sum_{j=1}^{m} j \bar{N}_{j}\left(p_{0}\right)}{\sum_{j=1}^{m} \bar{N}_{j}\left(p_{0}\right)}
$$

where $j^{*}$ is the variable eigenvalue of the electric power grid investment risk.

The attributive degree of the electric power grid investment risk tending to adjacent risk evaluation grades can be judged according to $j^{*}$. 


\subsection{The Framework of Risk Evaluation of Electric Power Grid Investment}

In this paper, the hybrid MCDM method for risk evaluation of electric power grid investment is proposed combining the BBWM and IMEEM. The BBWM, a latest group MCDM method, is adopted to calculate the weights of risk criteria which can consider the preferences of more than one decision makers, and the weight determination for risk criteria of electric power grid investment is more practical and credible. The IMEEM is used to rank the comprehensive risk grade of electric power grid investment, which uses the proximity degree rule other than maximum membership degree rule, and it can avoid evaluation information loss. Therefore, the risk ranking result of electric power grid investment by using the proposed hybrid MCDM method containing the BBWM and IMEEM is more appropriate and effective.

The detailed procedure of the hybrid novel MCDM method proposed in this paper for risk evaluation of electric power grid investment is shown in Figure 3.

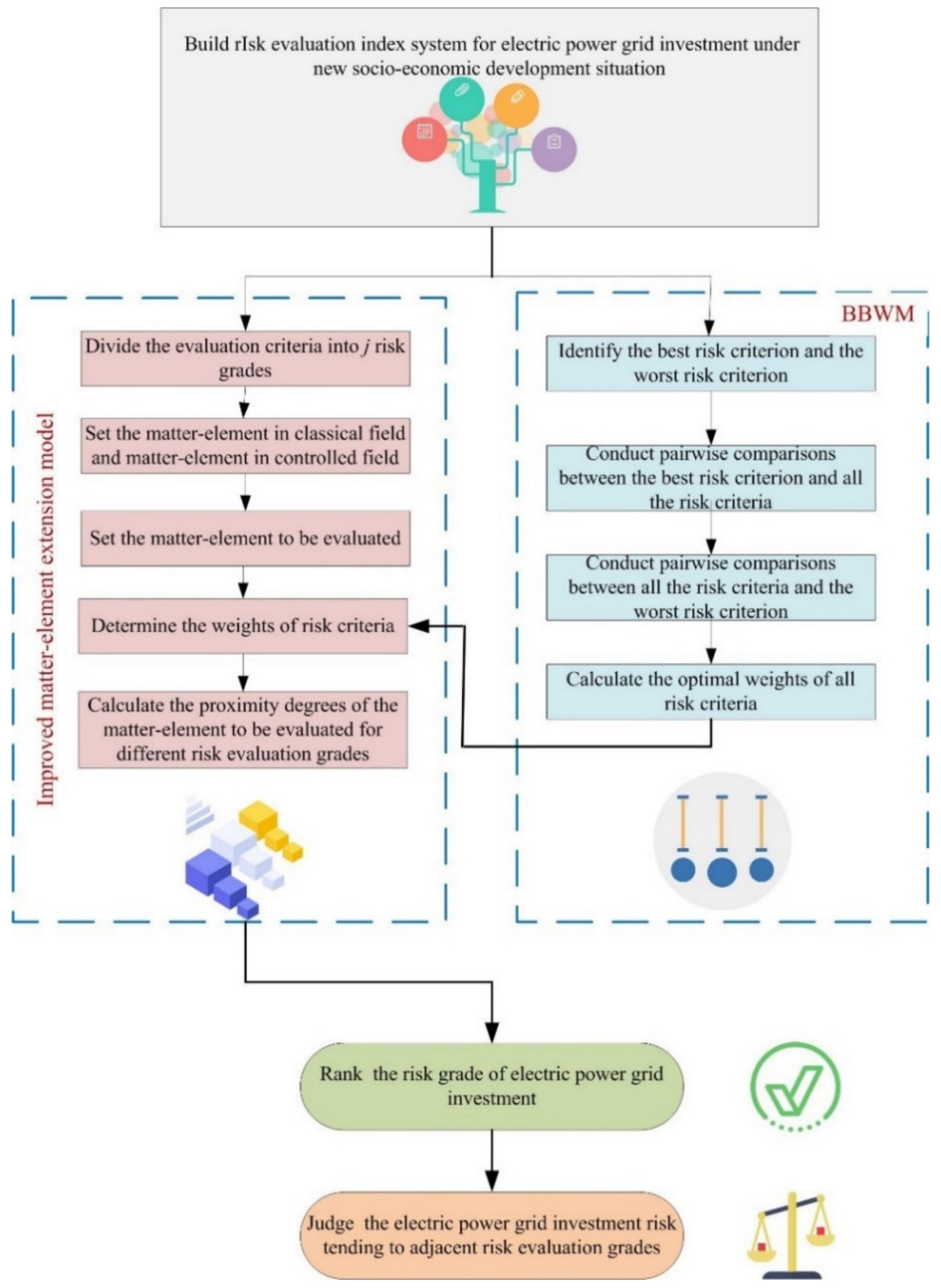

Figure 3. The procedure of the proposed hybrid novel multi-criteria decision making (MCDM) method for risk evaluation of electric power grid investment. 


\section{Empirical Analysis}

In China, there is a new socio-economic development situation currently. The risk of electric power grid investment in Inner Mongolia Autonomous Region of China will be evaluated in this section by using the hybrid MCDM method including the BBWM and IMEEM. The detailed steps are as follows.

\subsection{Set the Matter-Element for the Risk Evaluation of Electric Power Grid Investment}

According to the basic theory of the matter-element extension model, it can be known that the risk of electric power grid investment is the matter-element in this study. Meanwhile, the risk of electric power grid investment is divided into five grades, namely very low $(\mathrm{VL})$, low $(\mathrm{L})$, medium $(\mathrm{M})$, high $(\mathrm{H})$, very high $(\mathrm{VH})$. Therefore, it can be learnt that the matter-elements in the classical field are $R_{1}, R_{2}, R_{3}, R_{4}, R_{5}$, and there are $P_{1}, P_{2}, P_{3}$, $P_{4}, P_{5}$, correspondingly. Of which, $P_{1}$ represents very low electric power grid investment risk, $P_{2}$ represents low electric power grid investment risk, $P_{3}$ represents medium electric power grid investment risk, $P_{4}$ represents high electric power grid investment risk, and $P_{5}$ represents very high electric power grid investment risk.

According to the risk evaluation index system of electric power grid investment showed in Figure 1, all the risk criteria and sub-criteria of electric power grid investment are qualitative. Therefore, a scoring system with 10-point scale is adopted in this study, and the risk sub-criteria values of the matter-elements in the classical field $R_{1}, R_{2}, R_{3}, R_{4}$, $R_{5}$ are, respectively, $0-2,2-4,4-6,6-8$ and 8-10 successively. Then, the matter-elements in the classical field can be obtained as:

\begin{tabular}{|c|c|c|c|c|c|c|c|c|c|c|c|c|c|c|c|}
\hline $1=$ & $\begin{array}{c}P_{1} \\
C 1 \\
\langle 0,2\rangle\end{array}$ & $\begin{array}{c}C 2 \\
\langle 0,2\rangle\end{array}$ & $\begin{array}{c}C 3 \\
\langle 0,2\rangle\end{array}$ & $\begin{array}{c}C 4 \\
\langle 0,2\rangle\end{array}$ & $\begin{array}{c}C 5 \\
\langle 0,2\rangle\end{array}$ & $\begin{array}{c}C 6 \\
\langle 0,2\rangle\end{array}$ & $\begin{array}{c}C 7 \\
\langle 0,2\rangle\end{array}$ & $\begin{array}{c}C 8 \\
\langle 0,2\rangle\end{array}$ & $\begin{array}{c}C 9 \\
\langle 0,2\rangle\end{array}$ & $\begin{array}{c}C 10 \\
\langle 0,2\rangle\end{array}$ & $\begin{array}{c}C 11 \\
\langle 0,2\rangle\end{array}$ & $\begin{array}{c}C 12 \\
\langle 0,2\rangle\end{array}$ & $\begin{array}{c}C 13 \\
\langle 0,2\rangle\end{array}$ & $\begin{array}{c}C 14 \\
\langle 0,2\rangle\end{array}$ & $\begin{array}{c}C 15 \\
\langle 0,2\rangle\end{array}$ \\
\hline $2=$ & $\begin{array}{c}P_{2} \\
C 1 \\
\langle 2,4\rangle\end{array}$ & $\begin{array}{c}C 2 \\
\langle 2,4\rangle\end{array}$ & $\begin{array}{c}C 3 \\
\langle 2,4\rangle\end{array}$ & $\begin{array}{c}C 4 \\
\langle 2,4\rangle\end{array}$ & $\begin{array}{c}C 5 \\
\langle 2,4\rangle\end{array}$ & $\begin{array}{c}C 6 \\
\langle 2,4\rangle\end{array}$ & $\begin{array}{c}C 7 \\
\langle 2,4\rangle\end{array}$ & $\begin{array}{c}C 8 \\
\langle 2,4\rangle\end{array}$ & $\begin{array}{c}C 9 \\
\langle 2,4\rangle\end{array}$ & $\begin{array}{c}C 10 \\
\langle 2,4\rangle\end{array}$ & $\begin{array}{c}C 11 \\
\langle 2,4\rangle\end{array}$ & $\begin{array}{c}C 12 \\
\langle 2,4\rangle\end{array}$ & $\begin{array}{c}C 13 \\
\langle 2,4\rangle\end{array}$ & $\begin{array}{c}C 14 \\
\langle 2,4\rangle\end{array}$ & $\begin{array}{c}C 15 \\
\langle 2,4\rangle\end{array}$ \\
\hline$=$ & $\begin{array}{c}P_{3} \\
C 1 \\
\langle 4,6\rangle\end{array}$ & $\begin{array}{c}C 2 \\
\langle 4,6\rangle\end{array}$ & $\begin{array}{c}C 3 \\
\langle 4,6\rangle\end{array}$ & $\begin{array}{c}C 4 \\
\langle 4,6\rangle\end{array}$ & $\begin{array}{c}C 5 \\
\langle 4,6\rangle\end{array}$ & $\begin{array}{c}C 6 \\
\langle 4,6\rangle\end{array}$ & $\begin{array}{c}C 7 \\
\langle 4,6\rangle\end{array}$ & $\begin{array}{c}C 8 \\
\langle 4,6\rangle\end{array}$ & $\begin{array}{c}C 9 \\
\langle 4,6\rangle\end{array}$ & $\begin{array}{c}C 10 \\
\langle 4,6\rangle\end{array}$ & $\begin{array}{c}C 11 \\
\langle 4,6\rangle\end{array}$ & $\begin{array}{c}C 12 \\
\langle 4,6\rangle\end{array}$ & $\begin{array}{c}C 13 \\
\langle 4,6\rangle\end{array}$ & $\begin{array}{c}C 14 \\
\langle 4,6\rangle\end{array}$ & $\begin{array}{c}C 15 \\
\langle 4,6\rangle\end{array}$ \\
\hline$=$ & $\begin{array}{c}P_{4} \\
C 1 \\
\langle 6,8\rangle\end{array}$ & $\begin{array}{c}C 2 \\
\langle 6,8\rangle\end{array}$ & $\begin{array}{c}C 3 \\
\langle 6,8\rangle\end{array}$ & $\begin{array}{c}C 4 \\
\langle 6,8\rangle\end{array}$ & $\begin{array}{c}C 5 \\
\langle 6,8\rangle\end{array}$ & $\begin{array}{c}C 6 \\
\langle 6,8\rangle\end{array}$ & $\begin{array}{c}C 7 \\
\langle 6,8\rangle\end{array}$ & $\begin{array}{c}C 8 \\
\langle 6,8\rangle\end{array}$ & $\begin{array}{c}C 9 \\
\langle 6,8\rangle\end{array}$ & $\begin{array}{c}C 10 \\
\langle 6,8\rangle\end{array}$ & $\begin{array}{c}C 11 \\
\langle 6,8\rangle\end{array}$ & $\begin{array}{c}C 12 \\
\langle 6,8\rangle\end{array}$ & $\begin{array}{c}C 13 \\
\langle 6,8\rangle\end{array}$ & $\begin{array}{c}C 14 \\
\langle 6,8\rangle\end{array}$ & $\begin{array}{c}C 15 \\
\langle 6,8\rangle\end{array}$ \\
\hline$R_{5}=$ & $\begin{array}{c}P_{5} \\
C 1 \\
\langle 8,10\rangle\end{array}$ & $\begin{array}{c}C 2 \\
\langle 8,10\rangle\end{array}$ & $\begin{array}{c}C 3 \\
\langle 8,10\rangle\end{array}$ & $\begin{array}{c}C 4 \\
\langle 8,10\rangle\end{array}$ & $\begin{array}{c}C 5 \\
\langle 8,10\rangle\end{array}$ & $\begin{array}{c}C 6 \\
\langle 8,10\rangle\end{array}$ & $\begin{array}{c}C 7 \\
\langle 8,10\rangle\end{array}$ & $\begin{array}{c}C 8 \\
\langle 8,10\rangle\end{array}$ & $\begin{array}{c}C 9 \\
\langle 8,10\rangle\end{array}$ & $\begin{array}{c}\mathrm{C} 10 \\
\langle 8,10\rangle\end{array}$ & $\begin{array}{c}C 11 \\
\langle 8,10\rangle\end{array}$ & $\begin{array}{c}C 12 \\
\langle 8,10\rangle\end{array}$ & $\begin{array}{c}C 13 \\
\langle 8,10\rangle\end{array}$ & $\begin{array}{c}C 14 \\
\langle 8,10\rangle\end{array}$ & $\begin{array}{c}C 15 \\
\langle 8,10\rangle\end{array}$ \\
\hline
\end{tabular}

The matter-element in the controlled field $R_{p}$ is obtained as:

$R_{p}=\left[\begin{array}{ccccccccccccccc}P & & & & & & & & & & & & & & \\ C 1 & C 2 & C 3 & C 4 & C 5 & C 6 & C 7 & C 8 & C 9 & C 10 & C 11 & C 12 & C 13 & C 14 & C 15 \\ \langle 0,10\rangle & \langle 0,10\rangle & \langle 0,10\rangle & \langle 0,10\rangle & \langle 0,10\rangle & \langle 0,10\rangle & \langle 0,10\rangle & \langle 0,10\rangle & \langle 0,10\rangle & \langle 0,10\rangle & \langle 0,10\rangle & \langle 0,10\rangle & \langle 0,10\rangle & \langle 0,10\rangle & \langle 0,10\rangle\end{array}\right]^{T}$

\subsection{Set the Matter-Element to Be Evaluated}

According to the basic theory of the matter-element extension model, it can be known the matter-element to be evaluated is the risk of electric power grid investment under new socio-economic development situation in Inner Mongolia Autonomous Region of China. To obtain the performance values of fifteen risk sub-criteria in the risk evaluation index system of electric power grid investment, the expert panel including five experts in the fields of electric power industry management and enterprise risk management mentioned in Section 3 are also invited for valuing the performances of fifteen risk sub-criteria of electric power grid investment under new socio-economic development situation in Inner 
Mongolia Autonomous Region of China by using a scoring system with 10-point scale, and the results are shown in Figure 4.

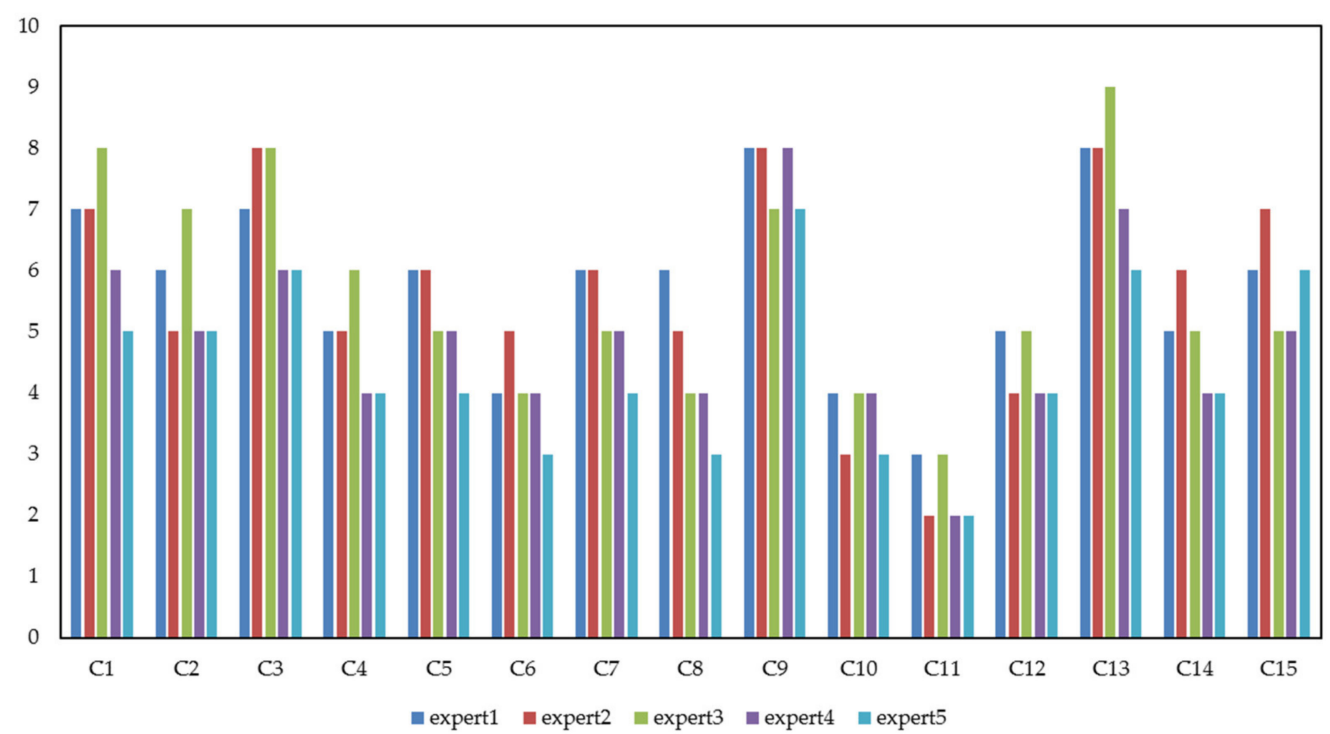

Figure 4. The performances of fifteen risk sub-criteria of electric power grid investment given by the five experts.

The final performance values of fifteen risk sub-criteria of electric power grid investment under new socio-economic development situation in Inner Mongolia Autonomous Region of China can be calculated by averaging the performance values of risk sub-criteria given by five experts. Then, the matter-element to be evaluated $R_{0}$ is obtained as:

$$
R_{0}=\left[\begin{array}{ccccccccccccccc}
P_{0} & & & & & & & & & & & & & & \\
C 1 & C 2 & C 3 & C 4 & C 5 & C 6 & C 7 & C 8 & C 9 & C 10 & C 11 & C 12 & C 13 & C 14 & C 15 \\
6.6 & 5.6 & 7 & 4.8 & 5.2 & 4 & 5.2 & 4.4 & 7.6 & 3.6 & 2.4 & 4.4 & 7.6 & 4.8 & 5.8
\end{array}\right]^{T} .
$$

It can be seen that the risk levels of different sub-criteria of electric power grid investment under new socio-economic development situation in Inner Mongolia Autonomous Region of China are quite different. The irresistible risk (such as nature disasters and COVID-19 pandemic) and electricity price fall risk are the highest, followed by economic downturn risk, financing risk, raw materials price increase risk, foreign exchange fluctuation risk, land acquisition risk, climatic condition risk, risk from inconformity between electric power plan and local development plan, delayed payback period risk, geographical condition risk, poor power grid engineering management risk, electricity policy fluctuation risk, power grid engineering quality risk, and the security incidents risk is the lowest. From the perspective of risk grade, it can be learnt that some sub-criteria risks belong to High grade (such as irresistible risk and electricity price fall risk), while some sub-criteria risks belong to Low grade (such as power grid engineering quality risk and security incidents risk). Therefore, it is difficult to evaluate the comprehensive risk level of electric power grid investment under new socio-economic development situation in Inner Mongolia Autonomous Region of China considering these fifteen risk sub-criteria together. Therefore, the MCDM technique is needed to be employed to comprehensively evaluate the risk level of electric power grid investment under new socio-economic development situation in Inner Mongolia Autonomous Region of China.

\subsection{Determine the Weights of Risk Sub-Criteria of Electric Power Grid Investment}

The BBWM is employed to calculate the weights of fifteen risk sub-criteria of electric power grid investment. The above-mentioned expert panel is also invited for the weight determination of fifteen risk sub-criteria of electric power grid investment. 
Firstly, five experts give the best risk sub-criterion and the worst risk sub-criterion of electric power grid investment, respectively. The results are tabulated in Table 1.

Table 1. The best risk sub-criterion and the worst risk sub-criterion given by expert panel.

\begin{tabular}{ccc}
\hline Expert Number & The Best Risk Sub-Criterion & The Worst Risk Sub-Criterion \\
\hline 1 & C 9 & C2 \\
2 & C15 213 & C 21 \\
3 & C13 & C11 \\
4 & C3 & C10 \\
5 & C & \\
\hline
\end{tabular}

Then, the experts conduct pairwise comparisons between the best risk sub-criterion and all other risk sub-criteria, which are listed in Table 2. Meanwhile, the experts also conduct pairwise comparisons between all other risk sub-criteria and the worst risk subcriterion, which are shown in Table 3.

Table 2. Pairwise comparisons conducted by expert panel for the best risk sub-criterion and all other risk sub-criteria.

\begin{tabular}{cccccc}
\hline Expert Number. & $\mathbf{1}$ & $\mathbf{2}$ & $\mathbf{3}$ & $\mathbf{4}$ & $\mathbf{5}$ \\
\hline The Best Risk Sub-Criterion & C9 & C15 & C13 & C3 & C9 \\
\hline C1 & 6 & 4 & 2 & 3 & 3 \\
C2 & 8 & 8 & 3 & 4 & 3 \\
C3 & 2 & 2 & 2 & 1 & 2 \\
C4 & 3 & 2 & 3 & 5 & 4 \\
C5 & 3 & 5 & 4 & 3 & 4 \\
C6 & 6 & 6 & 7 & 5 & 6 \\
C7 & 7 & 7 & 4 & 4 & 4 \\
C8 & 4 & 4 & 7 & 7 & 6 \\
C1 & 1 & 3 & 3 & 2 & 1 \\
C11 & 6 & 6 & 6 & 5 & 8 \\
C12 & 7 & 6 & 8 & 8 & 7 \\
C13 & 5 & 5 & 5 & 6 & 5 \\
C14 & 4 & 2 & 1 & 2 & 2 \\
C15 & 5 & 3 & 6 & 6 & 5 \\
\end{tabular}

Table 3. Pairwise comparisons between all other risk sub-criteria and the worst risk sub-criterion conducted by expert panel.

\begin{tabular}{cccccc}
\hline Expert Number & $\mathbf{1}$ & $\mathbf{2}$ & $\mathbf{3}$ & $\mathbf{4}$ & $\mathbf{5}$ \\
\hline The Worst Risk Sub-Criterion & C2 & C2 & C11 & C11 & C10 \\
\hline C1 & 3 & 5 & 7 & 6 & 5 \\
C2 & 1 & 1 & 6 & 4 & 5 \\
C3 & 7 & 7 & 7 & 8 & 7 \\
C4 & 7 & 6 & 5 & 4 & 5 \\
C5 & 6 & 3 & 5 & 6 & 4 \\
C6 & 4 & 3 & 2 & 4 & 2 \\
C7 & 3 & 2 & 4 & 5 & 4 \\
C8 & 6 & 2 & 2 & 3 \\
C9 & 8 & 5 & 6 & 7 & 8 \\
C10 & 3 & 3 & 3 & 3 & 1 \\
C11 & 2 & 1 & 1 & 2 \\
C12 & 2 & 4 & 3 & 3 \\
C13 & 5 & 4 & 7 & 6 \\
C14 & 7 & 3 & 2 & 3 \\
C15 & 5 & 5 & 5 & 6 \\
\hline
\end{tabular}


Then, the "Best-to-Others" vector $A_{B}$ can be obtained as:

$$
A_{B}=\left(\begin{array}{lllllllllllllll}
6 & 8 & 2 & 3 & 3 & 6 & 7 & 4 & 1 & 6 & 7 & 5 & 4 & 5 & 5 \\
4 & 8 & 2 & 2 & 5 & 6 & 7 & 4 & 3 & 6 & 6 & 5 & 2 & 3 & 1 \\
2 & 3 & 2 & 3 & 4 & 7 & 4 & 7 & 3 & 6 & 8 & 5 & 1 & 6 & 5 \\
3 & 4 & 1 & 5 & 3 & 5 & 4 & 7 & 2 & 5 & 8 & 6 & 2 & 6 & 4 \\
3 & 3 & 2 & 4 & 4 & 6 & 4 & 6 & 1 & 8 & 7 & 5 & 2 & 5 & 3
\end{array}\right)
$$

The "Others-to-Worst" vector $A_{W}$ can also be obtained as:

$$
A_{W}=\left[\begin{array}{lllllllllllllll}
3 & 1 & 7 & 7 & 6 & 4 & 3 & 6 & 8 & 3 & 2 & 5 & 5 & 4 & 5 \\
5 & 1 & 7 & 6 & 3 & 3 & 2 & 4 & 5 & 3 & 2 & 4 & 7 & 5 & 8 \\
7 & 6 & 7 & 5 & 5 & 2 & 4 & 2 & 6 & 3 & 1 & 4 & 8 & 3 & 3 \\
6 & 4 & 8 & 4 & 6 & 4 & 5 & 2 & 7 & 3 & 1 & 3 & 7 & 2 & 5 \\
5 & 5 & 7 & 5 & 4 & 2 & 4 & 3 & 8 & 1 & 2 & 3 & 6 & 3 & 6
\end{array}\right]^{T}
$$

Finally, the optimal weights' values of fifteen risk sub-criteria of electric power grid investment can be calculated using MATLAB software, and the results are shown in Figure 5.

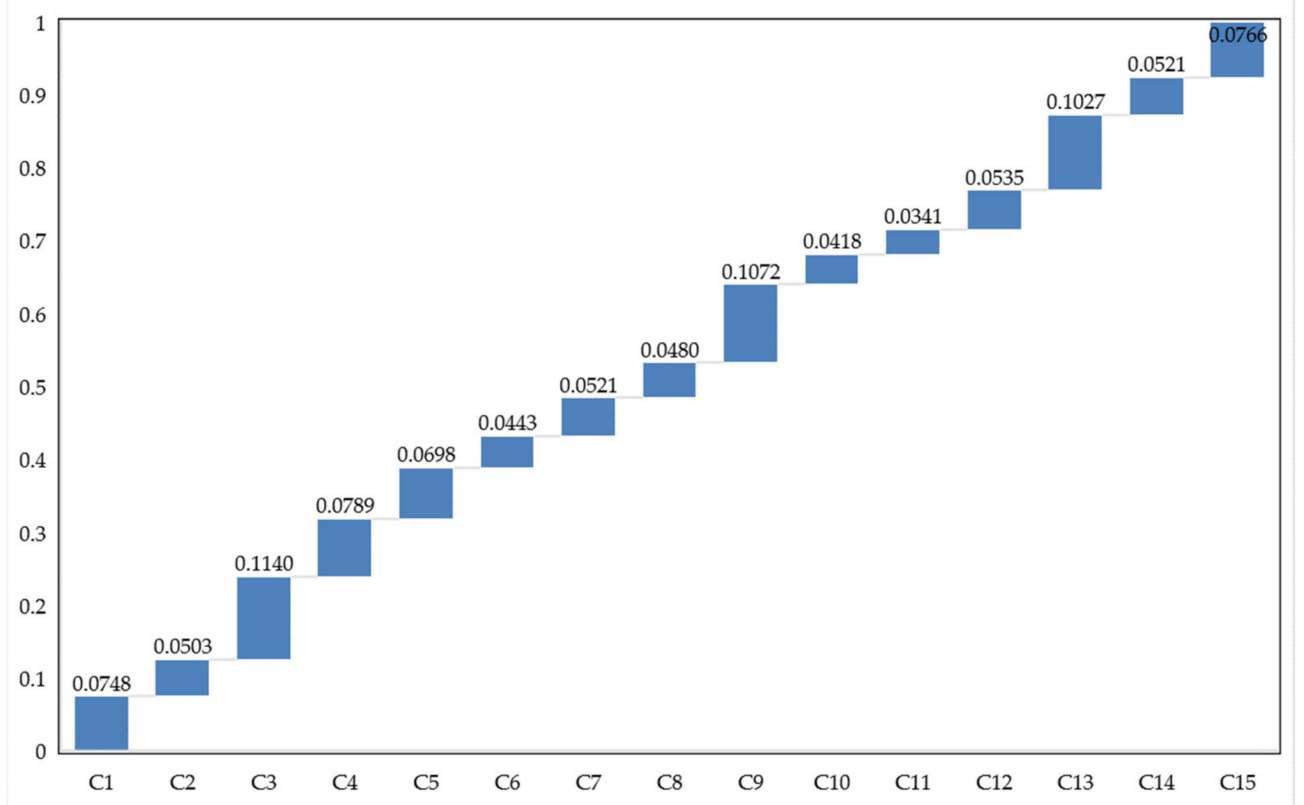

Figure 5. The weights of the fifteen risk sub-criteria of electric power grid investment.

It can be seen that the economic downturn risk sub-criterion (C3) is the most important risk sub-criterion among all the sub-criteria, followed by irresistible risk sub-criterion (C9), electricity price fall risk sub-criterion (C13), risk sub-criterion from inconformity between electric power plan and local development plan (C4), raw materials price increase risk sub-criterion (C15), financing risk sub-criterion (C1), land acquisition risk sub-criterion (C5), poor power grid engineering management risk sub-criterion (C12), climatic condition risk sub-criterion (C7), delayed payback period risk sub-criterion (C14), foreign exchange fluctuation risk sub-criterion (C2), geographical condition risk sub-criterion (C8), electricity policy fluctuation risk sub-criterion (C6), power grid engineering quality risk sub-criterion (C10), and security incidents risk sub-criterion (C11) is the least important risk sub-criterion among all the sub-criteria.

Figure 6 shows the creedal ranking of fifteen sub-criteria for risk evaluation of electric power grid investment under new socio-economic development situation in Inner Mongolia Autonomous Region of China, which shows the degree of certainty about the relations of fifteen risk sub-criteria. For instance, according to Figure 6, the irresistible risk 
sub-criterion (C9) is certainly more important than the security incidents risk sub-criterion (C11) with the confidence of 1 , and it is more desirable than raw materials price increase risk sub-criterion (C15) with the confidence of 0.92. According to this creedal ranking, it can be said that the weights' results of risk sub-criteria of electric power grid investment are effective and credible. Moreover, the ranking similarity for the weights rankings of these fifteen risk sub-criteria between each expert by using WS coefficient proposed by Wojciech Sałabun and Karol Urbaniak [42] can also be performed, which can verify the validity of risk sub-criteria weighting from another view.

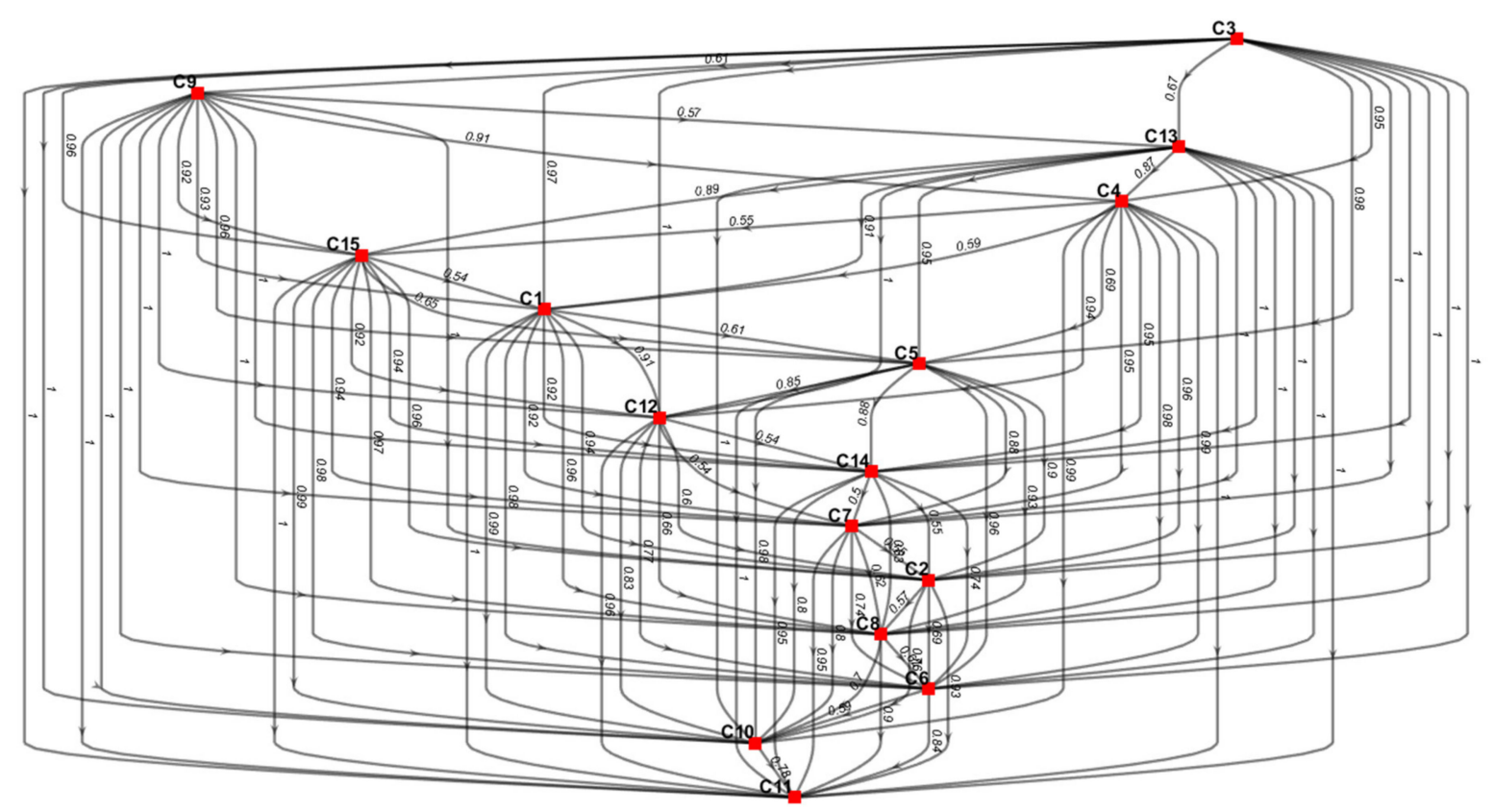

Figure 6. The credal ranking of fifteen risk sub-criteria for risk evaluation of electric power grid investment.

\subsection{Calculate the Proximity Degrees Related to Different Risk Evaluation Grades}

The proximity degrees of the risk of electric power grid investment under new socioeconomic development situation in Inner Mongolia Autonomous Region of China related to different risk evaluation grades including $\mathrm{VL}, \mathrm{L}, \mathrm{M}, \mathrm{H}$ and $\mathrm{VH}$ can be determined using Equation (9), which are:

$$
\begin{aligned}
& N_{1}\left(p_{0}\right)=1-\frac{1}{15 \times(15+1)} \sum_{i=1}^{15} D_{j}\left(v_{i}\right) w_{i}=0.9845, \\
& N_{2}\left(p_{0}\right)=1-\frac{1}{15 \times(15+1)} \sum_{i=1}^{15} D_{j}\left(v_{i}\right) w_{i}=0.9926, \\
& N_{3}\left(p_{0}\right)=1-\frac{1}{15 \times(15+1)} \sum_{i=1}^{15} D_{j}\left(v_{i}\right) w_{i}=0.9988, \\
& N_{4}\left(p_{0}\right)=1-\frac{1}{15 \times(15+1)} \sum_{i=1}^{15} D_{j}\left(v_{i}\right) w_{i}=0.9978, \\
& N_{5}\left(p_{0}\right)=1-\frac{1}{15 \times(15+1)} \sum_{i=1}^{15} D_{j}\left(v_{i}\right) w_{i}=0.9905 .
\end{aligned}
$$

where $N_{1}\left(p_{0}\right), N_{2}\left(p_{0}\right), N_{3}\left(p_{0}\right), N_{4}\left(p_{0}\right)$ and $N_{5}\left(p_{0}\right)$ represent the proximity degrees of the risk of electric power grid investment under new socio-economic development situation in Inner Mongolia Autonomous Region of China for $\mathrm{VL}, \mathrm{L}, \mathrm{M}, \mathrm{H}, \mathrm{VH}$ grades, respectively. 


\subsection{Risk Ranking of Electric Power Grid Investment}

According to Equation (10), it can be obtained that $N_{3}\left(p_{0}\right)=\max \left\{N_{j}\left(p_{0}\right)\right\}(j=1,2,3,4,5)$. Therefore, it can be concluded that the current risk grade of electric power grid investment under new socio-economic development situation in Inner Mongolia Autonomous Region of China is medium. According to Equations (11) and (12), it can be calculated that the variable eigenvalue $j^{*}=3.4143$. Therefore, it can be concluded that the current risk grade of electric power grid investment under new socio-economic development situation in Inner Mongolia Autonomous Region of China belongs to "Medium" grade and closer to the "High" grade. The result indicates although the current risk of electric power grid investment in Inner Mongolia Autonomous Region of China is not high, the future risk of electric power grid investment may become high, which should be paid more attention.

\section{Discussion}

In this section, the comprehensive risk of electric power grid investment in Inner Mongolia Autonomous Region of China obtained by the proposed hybrid MCDM method will be analyzed. Meanwhile, the comparative analysis of the proposed hybrid generalized MCDM method in this paper with the frequently used fuzzy comprehensive evaluation method and the sensitivity analysis will be also conducted in detail.

\subsection{Result Analysis}

The comprehensive risk of electric power grid investment in Inner Mongolia Autonomous Region of China is ranked by the proposed hybrid generalized MCDM method combining the BBWM and IMEEM. The ranking result indicates that the current comprehensive risk grade of electric power grid investment under new socio-economic development situation in Inner Mongolia Autonomous Region of China belongs to "Medium" grade and closer to the "High" grade. To obtain better insight from the proposed hybrid generalized MCDM method employed for comprehensive risk evaluation of electric power grid investment, it will probe into the performances and weights of risk sub-criteria.

Figure 7 shows the performances of these risk sub-criteria of electric power grid investment, and the weights of fifteen risk sub-criteria of electric power grid investment are displayed in Figure 8. According to Figure 7, it can be seen that nine of fifteen risk subcriteria have medium risks, which are raw materials price increase risk sub-criterion (C15), foreign exchange fluctuation risk sub-criterion (C2), land acquisition risk sub-criterion (C5), climatic condition risk sub-criterion (C7), risk sub-criterion from inconformity between electric power plan and local development plan (C4), delayed payback period risk subcriterion (C14), geographical condition risk sub-criterion (C8), poor power grid engineering management risk sub-criterion (C12), and electricity policy fluctuation risk sub-criterion (C6) in descending order. Four of fifteen risk sub-criteria have high risks, which are irresistible risk sub-criterion (C9), and electricity price fall risk sub-criterion (C13), economic downturn risk sub-criterion (C3), and financing risk sub-criterion (C1) in descending order. According to Figure 8, the weights of economic downturn risk sub-criterion (C3), irresistible risk sub-criterion (C9) and electricity price fall risk sub-criterion (C13) are more than 0.1 , the weights of risk sub-criterion from inconformity between electric power plan and local development plan (C4) and raw materials price increase risk sub-criterion (C15) are about 0.08 , and the weights of other medium risk are near or more than 0.05 . Therefore, it can be seen that most medium risk sub-criteria hold quite large weights, and meanwhile the high-risk sub-criteria also have large weights. Therefore, considering the above-mentioned risk sub-criteria performances and weights, the current comprehensive risk grade of electric power grid investment under new socio-economic development situation in Inner Mongolia Autonomous Region of China belongs to "Medium" grade, but it is much closer to the "High" grade. The irresistible risk sub-criterion, electricity price fall risk, economic downturn risk, financing risk and raw materials price increase risk should be paid more attention for the power grid company to prevent and control risks. 


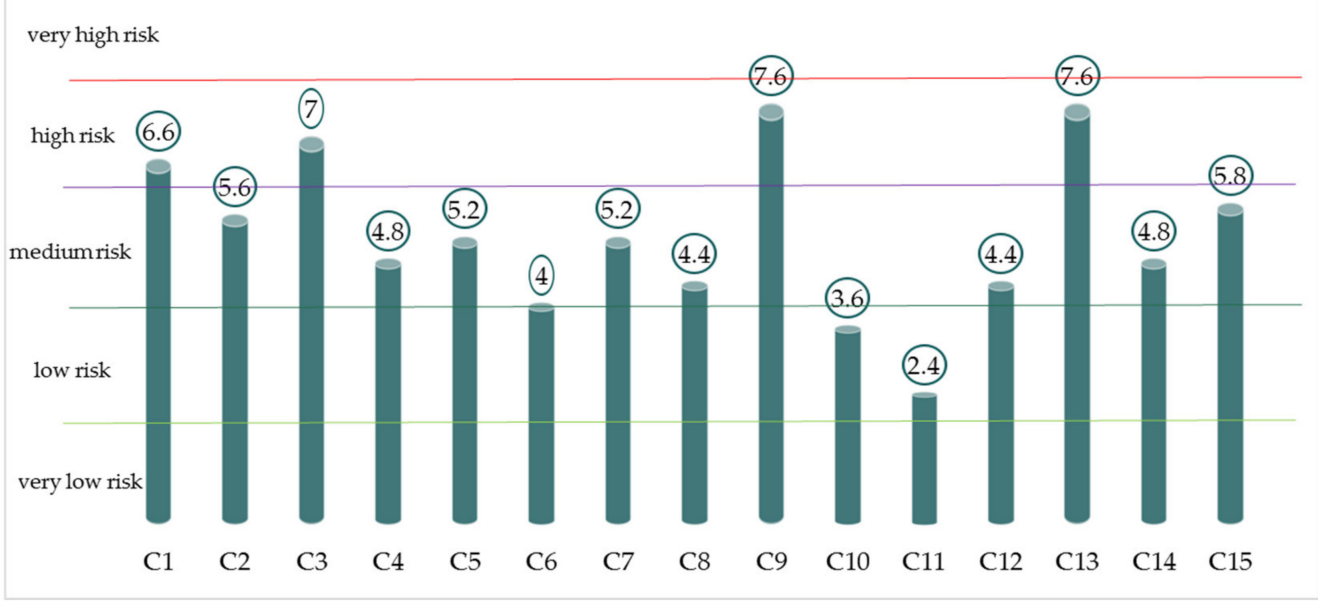

Figure 7. The risk sub-criteria performances of electric power grid investment.

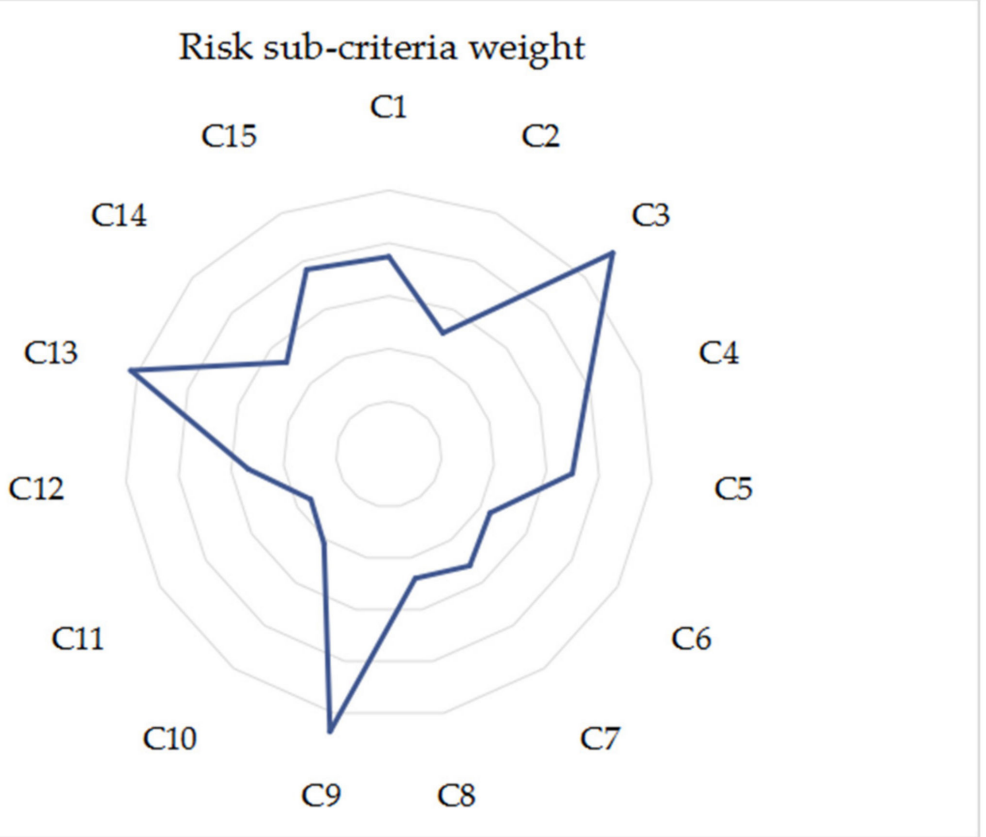

Figure 8. The weights of risk sub-criteria of electric power grid investment.

\subsection{Comparative Analysis}

The proposed hybrid generalized MCDM method combining the BBWM and IMEEM in this paper will be compared with the frequently used MCDM method, namely fuzzy comprehensive evaluation method. The fuzzy comprehensive evaluation method has been employed in many issues, and its basic theory can refer to Refs. [43-45].

For the risk evaluation of electric power grid investment under new socio-economic development situation in Inner Mongolia Autonomous Region of China by using the fuzzy comprehensive evaluation method, the risk grades are also set as the same as that of the proposed hybrid MCDM method in this paper, namely very low (VL), low (L), medium $(\mathrm{M})$, high $(\mathrm{H})$, very high $(\mathrm{VH})$. Meanwhile, similar to that in Section 5.1, a scoring system with 10-point scale is also adopted, and the risk sub-criteria values of five risk grades VL, L, M, H and VH are 0-2, 2-4, 4-6, 6-8 and 8-10, respectively. According to the performances 
(risk values) of fifteen risk sub-criteria of electric power grid investment given by the five experts shown in Figure 4, the fuzzy relation matrix $F$ can be obtained as follows:

$$
F=\left[\begin{array}{ccccc}
0 & 0 & 0.4 & 0.6 & 0 \\
0 & 0 & 0.8 & 0.2 & 0 \\
0 & 0 & 0.4 & 0.6 & 0 \\
0 & 0.4 & 0.6 & 0 & 0 \\
0 & 0.2 & 0.8 & 0 & 0 \\
0 & 0.8 & 0.2 & 0 & 0 \\
0 & 0.2 & 0.8 & 0 & 0 \\
0 & 0.6 & 0.4 & 0 & 0 \\
0 & 0 & 0 & 1 & 0 \\
0 & 1 & 0 & 0 & 0 \\
0.6 & 0.4 & 0 & 0 & 0 \\
0 & 0.6 & 0.4 & 0 & 0 \\
0 & 0 & 0.2 & 0.6 & 0.2 \\
0 & 0.4 & 0.6 & 0 & 0 \\
0 & 0 & 0.8 & 0.2 & 0
\end{array}\right]
$$

Then, the fuzzy comprehensive evaluation matrix $B$ can be calculated by combining $F$ and fifteen risk sub-criteria weights using the BBWM, which is:

$$
\left.\begin{array}{c}
B=\left(b_{1}, b_{2}, b_{3}, b_{4}, b_{5}\right)=w^{T} \circ F=\left(\begin{array}{c}
0.0748 \\
0.0503 \\
0.1140 \\
0.0789 \\
0.0698 \\
0.0443 \\
0.0521 \\
0.0480 \\
0.1072 \\
0.0418 \\
0.0341 \\
0.0535 \\
0.1027 \\
0.0521 \\
0.0766
\end{array}\right)^{T}\left[\begin{array}{ccccc}
0 & 0 & 0.4 & 0.6 & 0 \\
0 & 0 & 0.8 & 0.2 & 0 \\
0 & 0 & 0.4 & 0.6 & 0 \\
0 & 0.4 & 0.6 & 0 & 0 \\
0 & 0.2 & 0.8 & 0 & 0 \\
0 & 0.8 & 0.2 & 0 & 0 \\
0 & 0.2 & 0.8 & 0 & 0 \\
0 & 0.6 & 0.4 & 0 & 0 \\
0 & 0 & 0 & 1 & 0 \\
0 & 1 & 0 & 0 & 0 \\
0.6 & 0.4 & 0 & 0 & 0 \\
0 & 0.6 & 0.4 & 0 & 0 \\
0 & 0 & 0.2 & 0.6 & 0.2 \\
0 & 0.4 & 0.6 & 0 & 0 \\
0 & 0 & 0.8 & 0.2 & 0
\end{array}\right] . \\
=0.42310 .3075 \\
0.0205
\end{array}\right)
$$

According to the maximum membership degree principle, it can be deduced that $b_{3}=\max b_{i}(1 \leq i \leq 5)$. It can be seen that the comprehensive risk grade of electric power grid investment under new socio-economic development situation in Inner Mongolia Autonomous Region of China by using the fuzzy comprehensive evaluation method belong to "Medium".

Therefore, the comprehensive risk evaluation grade of electric power grid investment under new socio-economic development situation in Inner Mongolia Autonomous Region of China by using the proposed hybrid generalized MCDM method in this paper and the fuzzy comprehensive evaluation method is consistent. However, the proposed hybrid generalized MCDM method in this paper can obtain more ranking information, not only including the comprehensive risk grade, but also including the attributive degree to adjacent risk evaluation grades (this evaluation information cannot be obtained by using the fuzzy comprehensive evaluation method). Therefore, the proposed hybrid generalized MCDM method combining the BBWM and IMEEM in this paper for risk evaluation of electric power grid investment is effective and practical. 


\subsection{Sensitivity Analysis}

In this section, the sensitivity analysis focusing on the performances of fifteen risk subcriteria of electric power grid investment is conducted. Cases where the performances of fifteen risk sub-criteria of electric power grid investment have $10 \%, 20 \%$ and $30 \%$ less values and $10 \%, 20 \%$ and $30 \%$ more values than the base performances of fifteen risk sub-criteria are considered (namely, the performances of fifteen risk sub-criteria in Section 5). The sensitivity analysis result is shown in Figure 9. It can be seen that the $j^{*}$ will become larger with the increase of the performance values of fifteen risk sub-criteria of electric power grid investment, and it will become smaller with the decrease of the performance values of fifteen risk sub-criteria, but the $j^{*}$ is always in the interval $[3.30,3.50]$. Therefore, the result of sensitivity analysis related to the performances of fifteen risk sub-criteria of electric power grid investment indicates the current risk grade of electric power grid investment under new socio-economic development situation in Inner Mongolia Autonomous Region of China always belongs to "Medium" grade and closer to the "High" grade, which verifies the robustness of risk evaluation result of electric power grid investment in this paper.

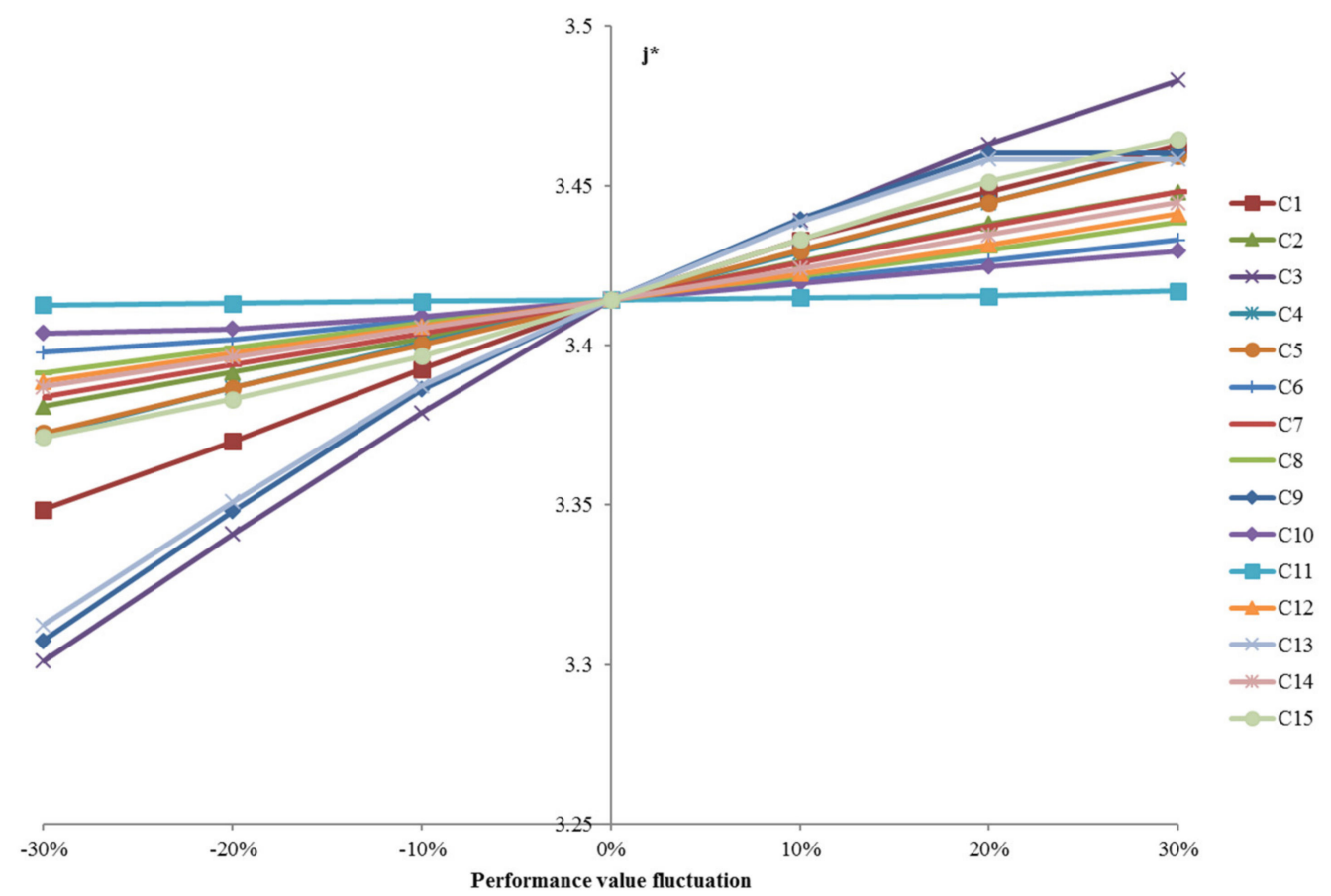

Figure 9. Sensitivity analysis result.

\section{Conclusions}

Electric power grid investment is very important to the economic development and energy transformation. In the past few years, China is undergoing new socio-economic development situation. The new-round marketization reform of electricity industry has been ongoing in China since 2015, and the recently proposed carbon peak and carbon neutral targets, new infrastructure development strategy and the COVID-19 pandemic have posed great impacts on the electric power grid investment in China. It can be said the electric power grid investment in China is facing more opportunities and challenges, which has also faced more risks. Therefore, it is very important to evaluate the risk of electric power grid investment in China under the new socio-economic development situation, which can aid the electric power grid managers to identify the risk and reduce risk loss, finally promote the sustainable development of electric power grid investment.

In this paper, a hybrid generalized MCDM framework for risk evaluation of electric power grid investment under new socio-economic development situation in China is proposed combining the BBWM for weights determination of risk criteria and the IMEEM for 
comprehensive risk grade rank of electric power grid investment. The empirical analysis focusing on the risk of electric power grid investment under new socio-economic development situation in Inner Mongolia Autonomous Region of China is performed, and the result indicates that the current risk grade of electric power grid investment under new socio-economic development situation in Inner Mongolia Autonomous Region of China belongs to "Medium". Meanwhile, the value of variable eigenvalue indicates that current risk level of electric power grid investment under new socio-economic development situation in Inner Mongolia Autonomous Region of China is closer to the "High" grade, which implies the risk of electric power grid investment under new socio-economic development situation in Inner Mongolia Autonomous Region of China will increase in the future, especially under the situation of COVID-19 pandemic and electricity marketization reforms.

Although it is verified that the proposed hybrid generalized MCDM method for risk evaluation of electric power grid investment under new socio-economic development situation in China is feasible and applicable, considering about the complexity and uncertainty of electricity marketization reform and socio-economic development situation, the risk evaluation index system of electric power grid investment can be updated in the future. In this paper, the important risk factors have been identified based on the weights and performances of risk sub-criteria, such as the irresistible risk, electricity price fall risk, economic downturn risk, financing risk and raw materials price increase risk, which should be paid more attention. The risk handling measurement for electric power grid investment under new socio-economic development situation in China will be studied in depth in the following research, which is a limitation in this paper. Meanwhile, the proposed hybrid generalized MCDM method does not consider the fuzziness of decision makers, which is another limitation of this paper. In the following research, a fuzzy MCDM method will be developed for risk evaluation of electric power grid investment. However, the hybrid generalized MCDM method proposed in this paper can also be used for electric power grid investment in other provinces in China and risk evaluation in other issues such as the electricity market and energy market.

Author Contributions: Conceptualization, S.G.; Data curation, Y.D. and Q.D.; Formal analysis, Y.S., X.F. and Q.D.; Funding acquisition, S.G.; Investigation, Y.Z.; Methodology, Y.D. and S.G.; Project administration, S.G.; Resources, Y.S., Y.Z. and X.F.; Software, Y.S. and X.F.; Visualization, Y.Z. and Q.D.; Writing—original draft, Y.D. and Y.S.; Writing—review and editing, S.G. All authors have and agreed to the published version of the manuscript.

Funding: This research was funded by Natural Science Foundation of China (grant number 71801092) and the Economic and Technological Research Institute of National Electric Power Grid Inner Mongolia Eastern Electric Power Co. Ltd. (grant number SGMDJY00JJJS2000066).

Institutional Review Board Statement: Not applicable.

Informed Consent Statement: Not applicable.

Data Availability Statement: Not applicable.

Acknowledgments: This research was funded by Natural Science Foundation of China (grant number 71801092) and the Economic and Technological Research Institute of National Electric Power Grid Inner Mongolia Eastern Electric Power Co. Ltd. (grant number SGMDJY00JJJS2000066). The authors are grateful to the editor and anonymous reviewers for their work.

Conflicts of Interest: The authors declare no conflict of interest.

\section{References}

1. Zhao, H.R.; Guo, S.; Zhao, H.R. Comprehensive assessment for battery energy storage systems based on fuzzy-MCDM considering risk preferences. Energy 2019, 168, 450-461. [CrossRef]

2. Berizzi, A.; Delfanti, M.; Falabretti, D.; Mandelli, S.; Merlo, M. Electrification Processes in Developing Countries: Grid Expansion, Microgrids, and Regulatory Framework. Proc. IEEE 2019, 107, 1981-1994. [CrossRef]

3. Yang, F.; Zhang, S.; Sun, C.W. Energy infrastructure investment and regional inequality: Evidence from China's power grid. Sci. Total Environ. 2020, 749, 10. [CrossRef] 
4. Parag, Y.; Ainspan, M. Sustainable microgrids: Economic, environmental and social costs and benefits of microgrid deployment. Energy Sustain Dev. 2019, 52, 72-81. [CrossRef]

5. Han, P.; Kimura, F.; Sandu, S. Household-level analysis of the impacts of electricity consumption on welfare and the environment in Cambodia: Empirical evidence and policy implications. Econ. Model. 2020, 89, 476-483. [CrossRef]

6. Zeng, M.; Peng, L.L.; Fan, Q.N.; Zhang, Y.J. Trans-regional electricity transmission in China: Status, issues and strategies. Renew. Sust. Energ. Rev. 2016, 66, 572-583.

7. Zhao, H.R.; Guo, S.; Zhao, H.R. Provincial energy efficiency of China quantified by three-stage data envelopment analysis. Energy 2019, 166, 96-107. [CrossRef]

8. Yao, Y.T.; Gao, C.W.; Tian, H.J.; Zhang, H.L. Review of mid-to long-term trading mechanism for renewable electricity consumption in Ningxia, China. Renew. Sust. Energ. Rev. 2020, 134, 23. [CrossRef]

9. Zeng, M.; Yang, Y.Q.; Wang, L.H.; Sun, J.H. The power industry reform in China 2015: Policies, evaluations and solutions. Renew. Sust. Energ. Rev. 2016, 57, 94-110. [CrossRef]

10. Zhao, H.R.; Zhao, H.R.; Guo, S. Comprehensive Performance Evaluation of Electricity Grid Corporations Employing a Novel MCDM Model. Sustainability 2018, 10, 23. [CrossRef]

11. Guo, S.; Zhang, W.Y.; Gao, X. Business Risk Evaluation of Electricity Retail Company in China Using a Hybrid MCDM Method. Sustainability 2020, 12, 21. [CrossRef]

12. Guindon, A.A.; Wright, D.J. Analytical approach to quantitative risk assessment for solar power projects. Renew. Sust. Energ. Rev. 2020, 133, 8. [CrossRef]

13. Chebotareva, G.; Strielkowski, W.; Streimikiene, D. Risk assessment in renewable energy projects: A case of Russia. J. Clean Prod. 2020, 269, 12. [CrossRef]

14. Sisodia, G.S.; Awad, E.; Alkhoja, H.; Sergi, B.S. Strategic business risk evaluation for sustainable energy investment and stakeholder engagement: A proposal for energy policy development in the Middle East through Khalifa funding and land subsidies. Bus. Strateg. Environ. 2020, 29, 2789-2802. [CrossRef] [PubMed]

15. Xu, L.; Tang, S.Y.; Deng, Y.F.; Yuan, Y. Sustainable operation-oriented investment risk evaluation and optimization for renewable energy project: A case study of wind power in China. Ann. Oper. Res. 2020, 290, 223-241.

16. Zhou, S.; Yang, P. Risk management in distributed wind energy implementing Analytic Hierarchy Process. Renew. Energy 2020, 150, 616-623. [CrossRef]

17. Adiyeke, E.; Canakoglu, E.; Agrali, S. Risk averse investment strategies for a private electricity generating company in a carbon constrained environment. J. Oper. Res. Soc. 2019, 70, 2056-2068. [CrossRef]

18. Felling, T.; Leisen, R.; Podewski, C.; Weber, C. Evaluation of Risks for Electricity Generation Companies through Reconfiguration of Bidding Zones in Extended Central Western Europe. Energy J. 2019, 40, 81-104.

19. Wu, Y.N.; Wang, J.; Ji, S.Y.; Song, Z.X. Renewable energy investment risk assessment for nations along China's Belt \& Road Initiative: An ANP-cloud model method. Energy 2020, 190, 19.

20. Yuan, J.H.; Li, X.Y.; Xu, C.B.; Zhao, C.H.; Liu, Y.X. Investment risk assessment of coal-fired power plants in countries along the Belt and Road initiative based on ANP-Entropy-TODIM method. Energy 2019, 176, 623-640. [CrossRef]

21. Kul, C.; Zhang, L.; Solangi, Y.A. Assessing the renewable energy investment risk factors for sustainable development in Turkey. J. Clean Prod. 2020, 276, 14. [CrossRef]

22. Yuan, J.H.; Zeng, Y.R.; Guo, X.X.; Ai, Y.; Xiong, M.P. Electric Power Investment Risk Assessment for Belt and Road Initiative Nations. Sustainability 2018, 10, 21. [CrossRef]

23. Egli, F. Renewable energy investment risk: An investigation of changes over time and the underlying drivers. Energy Policy 2020, 140, 15. [CrossRef]

24. Williams, N.J.; Jaramillo, P.; Taneja, J. An investment risk assessment of microgrid utilities for rural electrification using the stochastic techno-economic microgrid model: A case study in Rwanda. Energy Sustain Dev. 2018, 42, 87-96. [CrossRef]

25. Gregory, J.; Sovacool, B.K. The financial risks and barriers to electricity infrastructure in Kenya, Tanzania, and Mozambique: A critical and systematic review of the academic literature. Energy Policy 2019, 125, 145-153. [CrossRef]

26. Sałabun, W.; Jarosaw, W.; Shekhovtsov, A. Are MCDA Methods Benchmarkable? A Comparative Study of TOPSIS, VIKOR, COPRAS, and PROMETHEE II Methods. Symmetry 2020, 12, 1549. [CrossRef]

27. Kizielewicz, B.; Sałabun, W. A New Approach to Identifying a Multi-Criteria Decision Model Based on Stochastic Optimization Techniques. Symmetry 2020, 12, 1551. [CrossRef]

28. Guo, S.; Zhao, H.R. Optimal site selection of electric vehicle charging station by using fuzzy TOPSIS based on sustainability perspective. Appl. Energy 2015, 158, 390-402. [CrossRef]

29. Mohammadi, M.; Rezaei, J. Bayesian best-worst method: A probabilistic group decision making model. Omega-Int. J. Manag. Sci. 2020, 96, 8. [CrossRef]

30. Rezaei, J. Best-worst multi-criteria decision-making method. Omega-Int. J. Manag. Sci. 2015, 53, 49-57. [CrossRef]

31. Guo, S.; Zhao, H.R. Fuzzy best-worst multi-criteria decision-making method and its applications. Knowl.-Based Syst. 2017, 121, 23-31. [CrossRef]

32. Tan, Q.K.; Wei, T.; Peng, W.; Yu, Z.; Wu, C.R. Comprehensive evaluation model of wind farm site selection based on ideal matter element and grey clustering. J. Clean. Prod. 2020, 272, 11. [CrossRef] 
33. Asghari, M.; Saray, R.K.; Neshat, E. Misfire detection of homogeneous charge compression ignition engines using matter-element extension theory and thermodynamic multi zone model. Environ. Prog. Sustain. Energy 2020, 39, 13. [CrossRef]

34. Liu, S.L.; Li, W.P. Indicators sensitivity analysis for environmental engineering geological patterns caused by underground coal mining with integrating variable weight theory and improved matter-element extension model. Sci. Total Environ. 2019, 686, 606-618. [CrossRef] [PubMed]

35. Li, H.Z.; Guo, S. External Economies Evaluation of Wind Power Engineering Project Based on Analytic Hierarchy Process and Matter-Element Extension Model. Math. Probl. Eng. 2013, 2013, 11. [CrossRef]

36. Li, N.; Wang, H. Analysis on the evaluation of sustainable utilization of water resources by extension evaluation method. Arab. J. Geosci. 2020, 13, 8.

37. Wang, W.Q.; Lyu, S.R.; Zhang, Y.D.; Ma, S.Q. A Risk Assessment Model of Coalbed Methane Development Based on the Matter-Element Extension Method. Energies 2019, 12, 30. [CrossRef]

38. Xiao, Q.; Wan, S.S.; Lu, F.C.; Li, S. Risk Assessment for Engagement in Sharing Economy of Manufacturing Enterprises: A MatterElement Extension Based Approach. Sustainability 2019, 11, 29. [CrossRef]

39. Cai, W. Extension theory and its application. Chin. Sci. Bull. 1999, 44, 1534-1548. [CrossRef]

40. Li, H.; Guo, S.; Tang, H.; Li, C. Comprehensive evaluation on power quality based on improved matter-element extension model with variable weight. Power Syst. Technol. 2013, 37, 653-659.

41. Zhang, X. The definition of product about fuzzy comprehensive evaluation methods based on closeness. J. Shandong Univ. Nat. Sci. 2004, 39, 25-29.

42. Sałabun, W.; Urbaniak, K. A new coefficient of rankings similarity in decision-making problems. In Computational Science-ICCS 2020; ICCS 2020. Lecture Notes in Computer Science; Springer: Cham, Switzerland, 2020; pp. 632-645.

43. Feng, S.; Xu, L.D. Decision support for fuzzy comprehensive evaluation of urban development. Fuzzy Sets Syst. 1999, 105, 1-12. [CrossRef]

44. Liang, Z.; Yang, K.; Sun, Y.; Yuan, J.; Zhang, H.; Zhang, Z. Decision support for choice optimal power generation projects: Fuzzy comprehensive evaluation model based on the electricity market. Energy Policy 2006, 34, 3359-3364. [CrossRef]

45. $\mathrm{Wu}, \mathrm{X}$; $\mathrm{Hu}, \mathrm{F}$. Analysis of ecological carrying capacity using a fuzzy comprehensive evaluation method. Ecol. Indic. 2020, 113, 106243. [CrossRef] 